\section{Auch bei allergischer Rhinitis NO messen?}

\author{
Die Messung des Stickstoffmonoxidgehalts der Atemluft ist eine \\ validierte Methode für die Bestimmung der Entzündungsaktivität bei \\ Asthma bronchiale. Ob sich NO auch als Entzündungsmarker bei \\ allergischer Rhinitis eignet, wurde jetzt untersucht.
}

$\mathrm{B}$ ei Patienten mit unbehandeltem Asthma bronchiale korreliert die NO-Konzentration der Ausatemluft mit der Anzahl der Eosinophilen in den unteren Atemwegen. Unter einer antiinflammatorischen Behandlung sinkt die NO-Konzentration parallel zum Rückgang der Entzündungszellen.

Niederländische Allergologen bezogen nun 20 polysensibilisierte Patienten mit einer klinisch stabilen, unbehandelten allergischen Rhinitis in eine Studie ein. Die Messung der NO-Konzentration der Ausatemluft (eNO) erfolgte in einem standardisierten Testdurchlauf mit Hilfe einer Chemolumizur gleichen Tageszeit über 21 Tage. Außerdem wurde der Effekt einer doppelblind und plazebokontrolliert durchgeführten nasalen Provokation auf die NO-Konzentration gemessen und mit dem Symptomscore korreliert.

Die Reproduzierbarkeit der standardisierten Messung war innerhalb eines Sieben-Tage-Zeitraums gut, nahm aber über die 21 Testtage etwas $a b$. Eine mögliche Begründung für diese Beobachtung könnten Schwankungen des Allergengehalts der Atemluft sein, die sich klinisch häufig gar nicht bemerkbar machen. neszenz-basierten Messung - jeweils

\title{
Pollen stören Examen
}

Eine saisonale allergische Rhinokonjunktivitis beeinträchtigt nicht nur die Lebensqualität, sondern auch die Prüfungsergebnisse, wenn Pollensaison und Examenstermine zusammenfallen.

$\mathrm{n}$ einer Fall-Kontroll-Studie untersuchte ein britisches Autorenteam an 13 Schulen insgesamt 1.834 Schüler im Alter von 15 bis 17 Jahren. Sie werteten die Ergebnisse der Halbjahresprüfungen im Winter und der Abschlussprüfungen im Frühsommer aus. Als Fälle wurden diejenigen definiert, die während der Prüfungen vor den Sommerferien in wenigstens einem Kernfach - Mathematik, Englisch oder Naturwissenschaften - um mindestens einen Punkt schlechter abgeschlossen hatten als im Winter. Alle Schüler mit stabilen Noten dienten als Kontrollen.

In Mathematik verschlechterten sich $7 \%$ der Schüler, in Englisch 15\% und in Naturwissenschaften 20\%. Für die Analyse ergaben sich damit 662 Fälle und 1.172 Kontrollen. Zwischen 38\% und $43 \%$ der Schüler hatten an den Examenstagen im Sommer Symptome einer allergischen Rhinitis, zwischen $9 \%$ und 23\% wandten eine entsprechende Medikation an. Alle Prüfungstage zusammengenommen berichteten fast zwei Drittel der Schüler über Rhinitissymptome, $37 \%$ hatten zumindest einmal ein symptomatisch wirkendes Medikament appliziert, 6\% ein sedierendes Antihistaminikum.

Unter den Jugendlichen mit verschlechterten Prüfungsergebnissen gab es signifikant mehr Schüler mit Rhinitissymptomen als unter den Schülern mit stabilen Ergebnissen (Odds Ratio [OR] $1,43, p=0,002)$. Auch die Einnahme sedierender Antihistaminika war bei den Schülern, die sich verschlechtert hatten, signifikant häufiger $(\mathrm{OR}$ 1,36, $\mathrm{p}=0,01)$. Schließlich gab es auch mehr Asthmatiker unter den Fällen als unter den Kontrollen. Die Effekte zeigten auch eine Dosis-Wirkungs-Beziehung: Je schwerer
Im Vergleich zu den mit Plazebo provozierten Patienten stiegen nach Allergenprovokation die klinischen Symptome signifikant an $(\mathrm{p}<0,001)$, die NO-Konzentration in der Ausatemluft fiel 20 Minuten nach der Provokation $\mathrm{ab}(\mathrm{p}=0,001)$. Sieben Stunden später war die NO-Konzentration in der Verumgruppe im Vergleich zur Plazebogruppe leicht erhöht, nach 24 Stunden erreichte der Unterschied zwischen den beiden Gruppen Signifikanz ( $p=0,04)$.

Fazit: Die Messung des NO-Gehalts der Ausatmluft ist bei Patienten mit allergischer Rhinitis als Marker für die nasale Entzündungsaktivität prinzipiell geeignet. Die Reproduzierbarkeit der Messung ist gut, nimmt aber im Verlauf von einigen Wochen ab.

Boot JD et al. Nasal nitric oxide: longitudinal reproducibility and the effects of a nasal allergen challenge in patients with allergic rhinitis. Allergy 2007; 62: 378-84

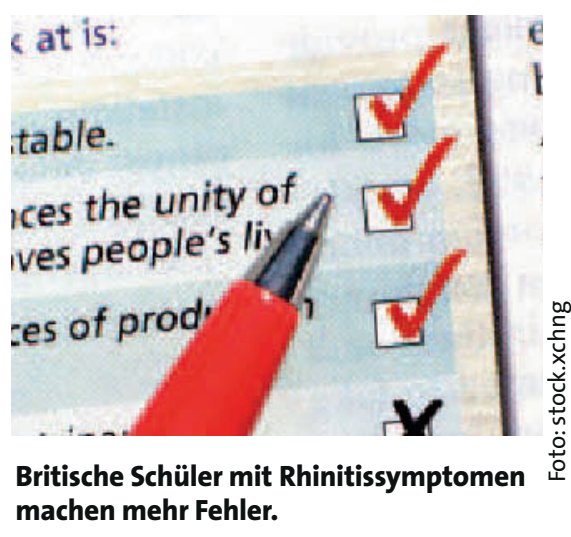

die Rhinitis-Symptomatik war, desto größer war die Wahrscheinlichkeit für eine Verschlechterung der Schulnoten.

Fazit: Schüler mit einer symptomatischen allergischen Rhinitis haben eine bis $\mathrm{zu}$ $40 \%$ erhöhte Wahrscheinlichkeit für schlechtere Schulnoten in der Pollensaison als ihre gesunden Kollegen. $\quad f k$

Walker $\mathbf{S}$ et al. Seasonal allergic rhinitis is associated with a detrimental effect on examination performance in United Kingdom teenagers: case-control study. J Allergy Clin Immunol 2007; 120: 381-7 